\title{
Flexibilización laboral en Argentina. Un camino hacia la precarización y la desocupación
}

\author{
Battistini, Osvaldo* \\ Montes Cató, Juan**
}

\section{Resumen}

El trabajo que presentamos aborda la problemática de la flexibilización laboral desde una perspectiva integral que recorre las diversas conceptualizaciones teóricas, el marco de la legislación laboral y la forma que obtuvo en los convenios colectivos. A fin de analizar como se instrumentaron dichas medidas, se realizo el estudio de casos en empresas de la actividad automotriz y de telecomunicaciones, centrando el análisis en la diferencia entre flexibilización formal e informal. Las hipótesis trabajadas son las siguientes: 1. La flexibilidad Laboral no contribuyó significativamente en el contexto en la cual se aplicó a disminuir los índices de ocupación y la informalidad; objetivo buscado por los diseñadores de politicas públicas; 2 . La flexibilidad laboral de hecho es más extensiva e intensiva que la de derecho; 3. A partir de las pautas flexibilizadoras se introducen cambios culturales substanciales que terminan por disolver las solidaridades internas de la clase trabajadora.

Palabras clave: Flexibilidad, desregulación, contrato colectivo, industria automotriz, telecomunicaciones.

Recibido: 99-10-15. Aceptado: 00-02-18

* Graduado en Clencias Políticas en la Universidad de Buenos Aires (UBA). Investigador del CElLPIETTE del CONICET. Docente de la UBA. Posee publicaciones en libros y revistas especializadas en el tema de relaciones laborales. Ha presentado variadas ponencias en Congresos Nacionales e internacionales.

* Graduado en Sociologia en la UBA. Becario del CONICET (con cede en el Programa de Investigaciones en Trabajo, Tecnología y Empleo CEIL-PIETTE). 


\title{
Labor Flexibility in Argentina. A Path Towards Labor Precariousness and Unemployment
}

\begin{abstract}
This paper touches on the problem of labor flexibility from an integral perspective and covers diverse theoretical concepts, the frame work for labor legislation, and the manner used to reach collective labor agreements. With the purpose of understanding how such agreements are reached, case studies were made in automotive and telecommunications industry firms, centering the analysis on the differences between formal and informal flexibility. The hypotheses worked with were the following: 1. Labor flexibility did not contribute significantly to lowering the rates of unemployment or of informal labor in the context of the situations where it was applied, which were the objectives desired by the designers of public policy; 2 . Labor flexibility is both more intensive and more extensive than labor law; 3. Labor flexibility introduces substantial cultural change that eventually dissolves worker class solidarity.
\end{abstract}

Key words: Flexibility, de-regulation, collective contracts, automobile industry, telecommunications industry.

\section{Introducción}

Desde la década de los '90 se sucedieron modificaciones profundas y estructurales en Argentina, modificaciones que si bien venlan esbozándose desde tiempo atrás, asumen un corpus homogéneo a partir de la Reforma del Estado, el proceso de Reestructuración Económica y Reconversión Productiva. Dichos procesos generaron cambios de orden jurídico, económico y social. Estas reformas impactaron significativamente en las relaciones laborales a través de la aplicación de la denominada "flexibilización laboral", asumida como una de las herramientas para llevar a cabo la adaptación de dichas relaciones al nuevo contexto productivo.
El trabajo que presentamos aborda la problemática de la flexibilización laboral desde una perspectiva que pretende integrar diversas dimensiones en el análisis del fenómeno comprendido. El desarrollo del estudio recorre las diversas conceptualizaciones teóricas, el marco de la legislación laboral y la forma que obtuvo expresión en los convenios colectivos. Vincular la implementación de la flexibilización laboral y el aumento del desempleo en Argentina, es el primer objetivo hacia el que se orienta el estudio.

El segundo objetivo, y a fin de analizar cómo se instrumentaron dichas medidas, consistió en el estudio de caso en empresas de la actividad automotriz y de telecomunicaciones, centrando el análisis en la diferencia entre flexibilización 
formal (flexibilidad de derecho, expresada en los convenios colectivos de trabajo especificos) e informal (flexibilidad de hecho, que refiere a las pautas flexibilizadoras no contempladas en los convenios colectivos, pero aplicadas en la práctica cotidiana de las empresas).

Las hipótesis trabajadas son las siguientes: 1. La flexibilidad laboral no contribuyó significativamente, en el contexto en la cual se la aplicó, a disminuir los indices de desocupación y la informalidad, objetivo buscado por los diseñadores de políticas públicas; 2 . La flexibilidad laboral de hecho es más extensiva e intensiva que la de derecho; 3 . A partir de las pautas flexibilizadoras se introducen cambios culturales substanciales que terminan por disolver las solidaridades internas de la clase trabajadora.

En primera instancia realizaremos un análisis conceptual sobre el término flexibilidad laboral; en segundo término analizaremos el comportamiento de la legislación argentina en relación a la flexibilización laboral y en tercer lugar estudiaremos la influencia de las transformaciones económicas y de la flexibilización laboral sobre las principales variables del mercado de trabajo, a través de la Encuesta Permanente de Hogares (EPH) ${ }^{1}$.
En cuarto término, mediante el estudio de dos casos particulares: actividad industrial, (metalúrgica-automotriz) y del sector servicios (telecomunicaciones) analizaremos el impacto en la organización del trabajo, considerando que ella se encuentra directamente relacionada con el modo de vida, y las modalidades de reproducción de los asalariados, según el análisis que realiza la escuela de la Regulación, cuando define el concepto relación salarial ${ }^{2}$.

\section{Apuntes para la conceptualización del término flexibilidad}

En la década de los '70 entra en crisis en los países desarrollados un modelo de acumulación basado en la organización del trabajo fordista y en las directrices keynesianas imbricadas con el Estado Benefactor. En 1978, lo que había sido visto como "simples turbulencias de una economia próspera" se expresó como una crisis de carácter estructural del modelo.

Los diversos enfoques que se constituyeron a fin de dar respuesta a dicha crisis estaban basados en el argumento que el modelo anterior habría acu-

1 La Encuesta Permanente de Hogares se realiza en dos oportunidades al año, sobre 28 aglomerados urbanos y es llevada a cabo por el Instituto Nacional de Estadisticas y Censos (INDEC).

La Escuela de la Regulación analiza el funcionamiento del régimen de acumulación a través de cinco formas institucionales, mediante las que se codifican una o varias relaciones fundamentales: la moneda, el estado, las formas de la competencia, las modalidades del régimen internacional y la relación salarial, siendo esta última un modo de "caracterizar las relaciones mutuas entre diferentes tipos de organización del trabajo, el modo de vida y las modalidades de reproducción de los asalariados" (Boyer, 1989). 
mulado excesivas rigideces en los mercados de productos, capitales y trabajo, acotando el margen en la acumulación del capital. Ante este escenario las respuestas que se ensayaron pivoteaban sobre la necesidad de disminuir la incidencia del estado sobre el mercado y el aparato productivo; a su vez otros enfoques sostenían que la caída de la rentabilidad provenia de las rigideces que se habían acumulado en los procesos de trabajo más que en una insuficiencia de la demanda global o de problemas de regulación del sistema.

Como sostiene Freyssinet (citado por Catalano; Hernández y Rojas, 1992), el núcleo que estaba en cuestionamiento era el acuerdo social entre los sindicatos y empresarios, acuerdo que actuaba como uno de los soportes del crecimiento económico. Ante este escenario, la flexibilidad laboral, se presenta para algunas escuelas como la "receta" adecuada para contener el aumento del desempleo, mejorar los estándares de competitividad $\mathrm{y}$, por último, como una medida para salvar la brecha generada por el empleo no registrado.

Un problema que surge, y al cual pretendemos aproximarnos en las líneas siguientes, refiere al contenido asignado al concepto de "flexibilización laboral". Si bien es utilizado generalizadamente, los atributos y características suelen hacer referencia a aspectos diversos, y en algunos casos contradictorios e incluso -fundamentalmente- las consecuencias de su implementación difieren radicalmente de un país a otro.

La cuestión central, tal como aquí está planteada, sugiere que las implicancias en cuanto al contenido del término no son homogéneas, por el contrario es utili- zado asignando diversos significados a un mismo significante. Esto resulta relevante si tomamos en cuenta el uso politi$c o$, en términos que quién se apropie del concepto, también posee la capacidad de asignarle aquellos atributos que favorezcan su posición. Por tal motivo, creemos que toda construcción conceptual se vincula fuertemente con el contexto en el cual se genera y principalmente en el cual se aplica; sin embargo, este argumento no inhibe la necesidad de constituir un panorama que facilite identificar las posibilidades con las cuales se cuenta.

Por tal motivo creemos pertinente, en primer lugar, realizar un mapéo de las diversas posturas desde el punto de vista teórico, a fin de construir cierta inteligibilidad al término.

J. Atkinson (citado por Santantonio y Tavilla, 1995) sostiene que existe una diferencia entre 1) la flexibilidad numéri$\mathrm{ca}$, que permite a las empresas adaptar el número de trabajadores, o el número de horas de trabajo, a los cambios de la demanda; 2) la flexibilidad funcional, como la capacidad de las empresas para reorganizar los puestos de trabajo de forma tal que los empleados puedan ejercer sus calificaciones realizando un mayor número de tareas; 3) el distanciamiento, el cual hace referencia a las subcontrataciones $y$ 4) las distintas formas de movilidad del colectivo de trabajo (geográfico, profesional y del empleo).

Por otra parte, A. Monza (citado por Santantonio y Tavilla, 1995) reconoce tres formas de flexibilidad que refieren a aspectos de índole contractual, tecnológica y salarial. La primera se encuentra orientada a la conformación de la relación salarial, vinculada con la estabilidad 
laboral y con algunos beneficios sociales; la segunda estaría asociada a las formas y modalidades organizacionales, a los cambios e innovaciones tecnológicos; el tercer aspecto asocia la remuneración salarial con la productividad de cada trabajador.

Boyer (1987) platea la diferenciación entre flexibilidad interna (ofensiva) y flexibilidad externa (defensiva). La flexibjlidad interna se refiere a elementos de la relación salarial vinculados a las modalidades y condiciones de puesta en práctica de la capacidad de trabajo. Multiprofesionalidad, polifuncionalidad, trabajo en equipos, implicación y mayor independencia en el trabajo, descentralización de las decisiones, carrera profesional, introducción de tecnología flexible, suelen ser sus componentes. En cuanto a la flexibilización externa, esta hace referencia a aquella que se desarrolló a partir de los ajustes de mercado, sostenida por la facilidad de entrada y salida de los trabajadores de las empresas sin redundar en costo alguno para el empresario (indemnizaciones, aportes sociales, etc.).

El mismo autor realiza un ejercicio de mayor descripción a fin de dilucidar los distintos aspectos que encierra el término y distingue cinco posibilidades de flexibilización: a) la capacidad de adaptar los equipamientos productivos a una demanda variable en volumen y en calidad, lo que constituye el "sistema de producción flexible". La flexibilidad se produce por adaptación interna a través de la incorporación de equipos reprogramables, de usos múltiples: máquinas herramientas de control numérico, robots, automatismos. En este aspecto Boyer incluye aquellas estrategias de externalización de la producción;

b) la predisposición de los trabajadores a cambiar de puesto de trabajo en el seno de su empresa, aún en situaciones de rápidas modificaciones de la organización del trabajo, lo que genera el "tipo" de agente de producción polivalente y móvil. Esta estrategia busca obtener de la fuerza de trabajo una mayor capacidad de respuesta a situaciones imprevistas y a exigencias de calidad;

c) la que implica el debilitamiento de las obligaciones legales de los empresarios respecto del contrato de trabajo, fundamentalmente en relación a la reglamentación que rige para los despidos ${ }^{3}$

d) la sensibilidad de los salarios (nominales y reales) para adaptarse a la situación económica general, condicionada por el mercado de trabajo; esta for-

En este mismo sentido sostienen Catalano; Hernández y Rojas (1992): "De modo general podría decirse que esta estrategia apunta en el extremo a una revisión diaria del contrato de trabajo para ajustar de modo permanente tanto el volumen como el tiempo y las condiciones de trabajo. Al interior de la misma se inscriben la eliminación de trabas legales a los despidos, formas de contratación por un cupo de horas anuales de trabajo que se acomodan a las necesidades y curvas de producción..." 
ma de flexibilización conduce a la aparición de salarios diferenciados y determinados por el rendimiento individual o por los resultados y los beneficios de cada empresa (productividad) y/o en función del desempleo. Su generalización tiende a introducir una fuerte diferenciación salarial por empresa y por productividad individual;

e) la posibilidad de las empresas de desentenderse de una parte relevante de sus obligaciones sociales (aportes jubilatorios, obras sociales, seguros sociales) y/o de eliminar o debilitar las normas jurídicas que regulan el contrato y su rescisión. La flexibilización socio-jurídica consiste en derogar o modificar parte de la legislación laboral y de la acción social del "Estado Benefactor", esta estrategia disminuye al mínimo la intervención del estado en la seguridad social.

Otras conceptualizaciones, hablan del modelo de flexibilidad "por lo alto", donde se contempla la posibilidad de lograr mayor eficiencia y calidad en la producción a partir de una situación de altos salarios, estabilidad del colectivo de trabajo y un sistema no autoritario de relaciones profesionales; básicamente apuntan a involucramiento de los trabajadores para con la empresa a través de células de trabajo, o trabajo en equipo. Esto se encuentra relacionado con sistemas productivos cercanos al modelo kalmariano, donde se exige una implicación de los trabajadores para obtener mejoras en la productividad y en la calidad de los productos.

Algunos autores vinculan la implementación de la flexibilidad laboral con las "instituciones del trabajo", de tal forma
Spyropoulos (1991) sostiene que la búsqueda de la adecuación del tiempo de trabajo y del número de trabajadores a la demanda es el núcleo central sobre el cual se sostiene dicha conceptualización. En este sentido, la implementación de la flexibilidad impactaria sobre las partes que conforman la relación laboral (empresas, sindicato y Estado) y su organización; las normas de regulación del mercado de trabajo; y la normativa relativa a la protección social y a la redistribución de los ingresos. El argumento propuesto se acerca a los sostenidos por aquellas posturas que asocian la flexibilidad laboral como una herramienta que apunta a destruir las bases sobre las que se sustenta el derecho del trabajo, porque se pretende abolir toda normativa tutelar.

El recorrido teórico no pretende ser exhaustivo, pero si presentar un panorama de las diversas posturas a las cuales se hace referencia cuando se pone en juego el concepto de flexibilización laboral. Cada una de ellas, podría plantearse, hace mayor énfasis en características económicas, sociales (desde el punto de vista de la organización) y legales. Desde la postura asumida en el presente trabajo, sostenemos que la definición más adecuada debería integrar las tres perspectivas citadas, ya que la flexibilidad laboral implica la puesta en juego de diversos factores que impactan en los tres niveles de análisis.

La perspectiva adoptada en el presente trabajo se vincula con la planteada por Boyer, ya que nos permite acercarnos al fenómeno teniendo en cuenta las tres dimensiones citadas (asumir una postura conceptual no inhibe futuras redefiniciones, en tanto y cuanto contribuyan al enri- 
quecimiento de la conceptualización). Por otra parte, posibilita una mejor operacionalización distinguiendo para cada caso en particular, qué aspecto es aplicado y cuáles son las implicancias que genera. Esto refiere a dos cuestiones: $1^{\circ} \mathrm{en}$ los casos concretos no necesariamente se aplica toda la bateria de posibilidades; $2^{\circ}$ el impacto de cada una es diferencial según el contexto en el cual se lo aplica.

En los apartados siguientes realizaremos un seguimiento por cada uno de las dimensiones antedichas, tratando de identificar la influencia de la flexibilización en la normativa, la situación social y económica de los trabajadores y el lugar que ocupan en la organización del trabajo.

\section{La flexibilidad en las transformaciones legislativas en Argentina}

En Argentina, la flexibilidad laboral acompañó la transformación del Estado en los comienzos de la puesta en marcha del nuevo régimen de acumulación.

La necesidad de adaptar la regulación normativa de las relaciones laborales a la nueva realidad productiva había comenzado a impregnar el discurso dominante a partir del planteo de la exigencia de eliminar las rigideces impuestas por la presión sindical sobre el Estado y las empresas. Aunque el discurso basado en la "modernización" venía desarrollándose desde mediados del gobierno radical, su puesta en práctica fue sólo posible a partir de las condiciones impuestas tras las crisis hiperinflacionarias de fines de la década de 1980 y principios de los '90.

El modelo económico puesto en funcionamiento mediante la Ley de Con- vertibilidad, las pautas impuestas por la reforma del Estado y la Ley de Emergencia Económica, sirvieron como marco a la implementación de medidas destinadas a desregular las relaciones laborales.

En 1991, amparándose en la necesidad de implementar medidas destinadas a controlar el aumento del desempleo y del número de trabajadores no registrados el parlamento promulgó la Ley Nacional de Empleo ( $\left.N^{\circ} 24.013\right)$. Mediante esta norma se promovia la utilización de formas contractuales, cuyas premisas se asentaban en el debilitamiento de los mecanismos de ingreso y egreso de los trabajadores a la empresa, a partir de las cuales se pretendia abandonar el paradigma de la relación salarial por tiempo indeterminado, pero se mantenian reglamentaciones que traducian mecanismos culturales enraizados en el sistema de relaciones laborales argentino.

Si bien la relación de fuerzas entre capital y trabajo comenzaba a presentar las condiciones para el desarrollo de mecanismos flexibilizadores y el abandono paulatino de las normativas aprobadas en el momento en que los sindicatos contaban con capacidad de presión suficiente sobre el Estado, ciertos parámetros culturales e institucionales impidieron su desarrollo en forma completa y en el corto o mediano plazo (Battistini, 1998).

La legitimidad que podía conseguirse a través del miedo al caos no alcanzaba para abrir a los empresarios la posibilidad de desprenderse de su dependencia a los sindicatos. En este sentido, se mantenía aún cierto control en manos de los sindicatos para la implementación de estas nuevas formas de contratación. Sólo podian ser aprobadas mediante los convenios co- 
lectivos de trabajo. La misma redacción de la ley quizás es el anticipo de las ambivalencias y contradicciones que el Estado presentó en el desarrollo de la flexibilidad en la legislación laboral.

A pesar de que la nueva norma estatal impone la introducción de las modalidades temporarias de contratación, comienza ratificando el principio de indeterminación del plazo como modalidad principal del contrato de trabajo y establece que en caso de duda respecto a la implementación de las mismas, serán consideradas por tiempo indeterminado. Además, en todos estos nuevos contratos se mantiene la condición de preavisar antes de la finalización del plazo y, en dos de ellos ${ }^{4}$ se impone la obligación del pago de indemnización al término de la relación.

La implementación de estos instrumentos debía ser funcional a los desafíos que planteaban las incipientes transformaciones en la organización de la producción, y al mismo tiempo se convertian en mecanismos que permitirian reducir el desempleo.

El Título II de la misma ley estaba destinado al control del empleo no registrado. En el mismo se planteaban sanciones para los empleadores que no registraran las relaciones laborales e indemnizaciones para los trabajadores bajo esa forma de contratación irregular. Sin embargo, la ley que imponía los castigos incluía, en su mismo articulado, disposiciones que otorgaba la posibilidad a los empleadores de eludirlas, mediante plazos de regularización por parte de los empre- sarios sin obligación del pago de las multas y las contribuciones no abonadas. Con estos mecanismos se daban señales contradictorias y se presentaba, de algún modo, la ambigüedad que acompañaría la política laboral del gobierno menemista en materia de reformas a la legislación.

En 1992, mediante el Decreto 340 , se aprobó el sistema de pasantias, mediante el cual se pretendía lograr una conexión entre la educación formal y la práctica laboral tanto para estudiantes como para docentes, con el fin de facilitar la integración de los mismos en el mundo laboral. La posterior implementación de estas pasantías derivó en formas de contratación mediante las cuales se eluden los derechos derivados de una relación salarial (impuestos a la seguridad social, vacaciones, aguinaldo, indemnización, etc.). Además, la ausencia de control por parte del Estado y las instituciones educativas (públicas y privadas) dio lugar a prácticas alejadas de los objetivos de la misma normativa, ya que no se cumple con la premisa de que la práctica laboral se ajuste a la formación previa.

Desde 1991 y después de varios intentos del Poder Ejecutivo para que el Parlamento apruebe distintos proyectos de reforma laboral, recién en 1995, y tras la firma del "Acuerdo Marco para la Productividad y el Empleo", lograron aprobarse dos nuevas leyes flexibilizadoras: el Nuevo Régimen de Contrato de Trabajo ( $N^{\circ} 24.465$ ) y el Régimen Laboral para las Pequeñas y Medianas Empresas ( $\left.\mathrm{N}^{\circ} 24.467\right)$. 
Mediante la primera de ellas se introdujeron en la Ley de Contrato de Trabajo, el periodo de prueba ${ }^{5}$, el contrato a tiempo parcial ${ }^{6}$ y dos nuevas modalidades temporarias de relación laboral ${ }^{7}$.

Las nuevas formas atipicas de contratación, sumadas a las ya existentes venían a reforzar la tendencia contradictoria de la política laboral desde 1991 en adelante. En la Ley Nacional de Empleo, el Poder Ejecutivo manifestaba su preocupación por la existencia y crecimiento de relaciones contractuales irregulares (empleo no registrado) pero, al mismo tiempo incorporaba normativas que legitimaban dichas relaciones (estas modalidades temporarias contaban con reducciones entre el $50 \%$ y el $100 \%$ en los impuestos a la seguridad social).

Mediante el Régimen Laboral para PYMES se otorga la posibilidad a estas empresas para utilizar las modalidades promovidas de contratación sin previa habilitación de los convenios colectivos de trabajo y en dos de ellas se las eximía del pago de la indemnización que debia abonarse, al final del periodo. Por medio de la misma norma se pretendía impulsar la negociación colectiva en este tipo de empresas, ya que se establecia la obligación de que todo convenio negociado por actividad industrial a partir de los seis meses de vigencia de la ley tenga un capítulo para las PyMES de dicha actividad. Esto no fue respetado por la misma autoridad de aplicación, ya que desde 1995 hasta mediados de 1999 sólo aproximadamente 20 convenios colectivos de actividad contaron con un capítulo específico.

En esta ley se avanzaba en dos viejas aspiraciones del sector empresario: la eliminación de la ultractividad ${ }^{8}$ de los convenios, por lo menos en este ámbito y la disponibilidad colectiva. En el primer caso se planteaba la extinción de los convenios de pleno derecho a los tres meses de su vencimiento, de no mediar estipulación en contrario.

El período de prueba como plazo previo a la contratación por tiempo indeterminado, con un plazo máximo de 3 meses. También se otorgaba la posibilidad de extender dicho periodo a 6 meses por medio de los convenios colectivos de trabajo.

6 El contrato a tiempo parcial, para aquellas relaciones laborales por tiempo indeterminado pactadas con una jornada inferior a los 2/3 de la jornada habitual de la actividad.

7 Se trata del contrato de aprendizaje, para jóvenes sin empleo entre 14 y 25 años en la cual no se establecia relación laboral entre trabajador y empleador; y la Modalidad Especial de Fomento del Empleo, para trabajadores mayores de 40 años, en la cual se reducian al $50 \%$ las contribuciones patronales y no se obligaba a los empleadores al pago del preaviso, la indemnización y se otorgaba la posibilidad de modificar, por medio de los convenios colectivos de trabajo, el derecho al pago de indemnización ante la ruptura del contrato por parte del empleador.

Según la ley que regula la negociación colectiva $\left(N^{\circ} 14.250\right)$, si se produce el vencimiento de un convenio y las partes no acuerdan la realización de uno nuevo, este seguirá vigente hasta que dicho acuerdo se produzca. De este modo, en Argentina, aún no se han modificado gran parte de las negociaciones formales llevadas a cabo en 1975. 
En el segundo caso, se otorgó una preeminencia a la negociación colectiva de pequeñas empresas, ya que esta última no podía ser afectada por convenios de ámbito superior. Además se dispuso que mediante dichas negociaciones se podia: a) modificar en cualquier sentido las formalidades, requisitos, aviso y oportunidad del goce de las vacaciones; b) disponer el fraccionamiento, en no más de tres períodos, el pago del sueldo anual complementario; c) modificar el régimen de extinción de los contratos de trabajo, incluso con la posibilidad de establecer un sistema de cuentas de capitalización individual. De este modo se abandonaba el principio protectorio de la norma de ámbito superior.

Otro de los artículos significativos de esta Ley estaba destinado a la eliminación de la integración del mes de preaviso en la indemnización, dado que pasaba a considerarse el mismo a partir del día siguiente a su comunicación. Además se dispuso que debía ser de no más de un mes cualquiera sea la antigüedad del trabajador en el empleo ${ }^{9}$.

Dado que se considera que aproximadamente el $80 \%$ del empleo corresponde a las PyMES, con esta nueva normativa especifica se pretendía otorgar facilidades a los empresarios para incrementar la contratación.
Con el impulso a la reforma económica que se plantea a partir de la Ley de Convertibilidad, se promulga el decreto 1334/91 en el que se imponen pautas especificas a tener en cuenta en la firma de cualquier acuerdo o convenio colectivo. Desde la promulgación de este decreto, toda negociación colectiva requeriria, para ser homologada por el Ministerio del Trabajo y de la Seguridad Social, que no se incluyeran cláusulas violatorias de normas de orden público dictadas en interés general, que no se establezcan mecanismos indexatorios prohibidos por la Ley $N^{\circ} 23.928$ (de convertibilidad), que la vigencia de las convenciones no afecte significativamente la situación económica general y que los posibles incrementos salariales sean acordados en función de pautas de productividad, eficiencia y racionalidad.

A partir de 1991, entonces, se introdujeron en los convenios colectivos, una serie de cláusulas que pueden ser calificadas como flexibilizadoras. La supuesta "productividad" encubrió la implementación de mecanismos destinados a la adaptación de las relaciones laborales a las necesidades de las actividades o las empresas. En muchos casos se alcanzó un mayor grado de flexibilidad que el obtenido mediante reformas a la legislación laboral. (Battistini, Montes Cato, 1998;

9 Para las relaciones contractuales que no corresponden a las PyMEs, hasta la reforma de 1998, el preaviso regía desde el primer día hábil del mes posterior a la comunicación al trabajador, por lo cual si esto se llevaba a cabo antes de finatizar el mes debía pagarse dicho lapso. Además, para el resto de las empresas el preaviso es de un mes si la antigüedad del trabajador es inferior a cinco años y de dos meses si es superior. 
Battistini, Jabbaz et. al, 1996; Deibe, Matheu et. al 1994).

En 1993, mediante el Decreto 470 , se establece que el nivel de una negociación podría ser modificado, a petición de cualquiera de las partes signatarias de un convenio colectivo. Mediante esta norma se impulso una cierta descentralización de la negociación a nivel de la empresa y su articulación con convenios de ámbito sectorial. Desde entonces, el mayor número de convenios colectivos fue realizado a nivel de empresa.

Finalmente, en 1998 se produce un viraje muy importante en la política desreguladora del gobierno. Muchas de las pautas que se habian privilegiado en el anterior proceso flexibilizador que se desarrollo hasta 1995 mediante las leyes aprobadas y hasta 1997 en los proyectos y decretos no aprobados, fueron abandonadas tras el reemplazo de Caro Figueroa por Erman González en el Ministerio de Trabajo. Privilegiando una mejor relación con la Confederación General del Trabajo y alentando la posibilidad de una nueva reelección del presidente Menem, se impulsó la aprobación de una ley que anuló anteriores pautas flexibilizadoras y estableció mecanismos destinados a asegurar el control sindical sobre la negociación colectiva.

Mientras en el proceso anterior se respetaban, sin mayores modificaciones, las pautas impuestas por el Fondo Monetario Internacional, en este nuevo período el gobierno enfrentó las posturas de los representantes de este organismo financiero internacional y las de las organizaciones empresariales para sostener un proyecto más cercano a las posiciones de la central sindical oficialista.
Así, después de duros debates en el Parlamento se logró aprobar la Ley $\mathrm{N}^{\circ}$ 25.013 , entre cuyos cambios más significativos podemos destacar los siguientes:

- Eliminación de las modalidades de contratación promovidas.

- Se reduce a 30 días el período de prueba, y se mantiene la posibilidad de extenderlos mediante la negociación colectiva a seis meses, pero en este caso de deben realizar todos los aportes y contribuciones a la seguridad social y rigen las normas referidas a la indemnización y el preaviso.

- Se modifica el régimen de extinción del contrato de trabajo, reduciendo las indemnizaciones por despido injustificado y por fuerza mayor y eliminando la integración del mes de preaviso.

- Se determina la caducidad de las convenciones colectivas que no hayan tenido negociaciones después del $1^{\circ}$ de enero de 1988.

- Se impone como representación de los trabajadores en la negociación colectiva a la asociación gremial de grado superior, la que podrá delegar el poder de negociación en sus propias estructuras descentralizadas.

Algunos de estos artículos fueron duramente cuestionados por los empresarios. Entre ellos se destaca la anulación de las modalidades promovidas de contratación, dado que para este sector constituian un recurso adecuado para regular sus planteles ante las diversas coyunturas económicas y escaparle a relaciones más estables con los trabajadores. 
Asimismo, las críticas se dirigieron al acortamiento en el plazo del período de prueba. Pero el cuestionamiento más significativo fue hacia la negativa del gobierno a anular la ultractividad de los convenios colectivos. Aunque la ley planteaba la caducidad de aquellos que no hayan tenido negociaciones posteriores a 1988, la mayor parte de los convenios habian pasado por alguna forma de negociación, considerando entre estas a los acuerdos salariales. Así, aún los convenios vigentes desde 1975 no perderian vigencia por haber negociado algún acuerdo especifico en la actividad, alguna rama o empresa.

También se incluye entre las reglamentaciones de esta ley el refuerzo de la centralización de la representación sindical en las negociaciones colectivas, mediante la prioridad de representación a la organización de nivel mayor. Esto fue criticado tanto por las organizaciones empresariales, porque se dificulta cada vez más la posibilidad de actividad del sindicato de empresa, como por algunas organizaciones sindicales, dado que se las obliga a depender de las organizaciones de ámbito superior en sus negociaciones descentralizadas (rama, región o empresa) ${ }^{10}$.

En el próximo apartado efectuamos un seguimiento de estas transformaciones en la legislación laboral respecto a la evolución de algunos indicadores del mercado laboral.

\section{Impacto de los cambios legales en el mercado de trabajo}

A comienzos de 1989 , en pleno proceso hiperinflacionario la tasa de desocupación habia alcanzado al $8 \%$ (mayo de 1989) en todos los aglomerados urbanos, índice que se repite en la medición de mayo del año siguiente. Entre estas dos mediciones se vivía el proceso de transición entre los dos primeros gobiernos democráticos, pero aún el menemismo en el poder no lograba recomponer las variables económicas y la hiperinflación era de nuevo el estigma que jaqueaba a los economistas de turno. Mientras tanto, ya se notaban signos de precarización en el mercado de trabajo, ya que la subocupación ascendía a valores cercanos al $9 \%$ (ver cuadro No. 1 ).

Junto al Plan de Convertibilidad, en 1991, el Poder Ejecutivo lanza dos normas laborales con especial influencia respecto a la flexibilización laboral: el Decreto 1334, que impone la negociación por productividad y la Ley Nacional de Empleo. Estas primeras medidas parecen tener un impacto positivo en las variables del mercado de trabajo, ya que se

10 Tras el cambio de gobierno, a fines de 1999, la flexibilidad laboral vuelve a ser el tema en discusión, retomando algunos de los puntos que habian sido modificados por esta última ley. El proyecto flexibilizador de la Alianza (compuesta por la Unión Civica Radical y el Frente Pais Solidario) propone el aumento del período de prueba a un año, la eliminación de la uitractividad y la descentralización total de la negociación colectiva. Cabe aclarar que algunos de estos items eran las exigencias planteadas por el FMI en el proceso anterior de reformas laborales. 


\begin{tabular}{|c|c|c|c|c|c|c|c|}
\hline \multicolumn{8}{|c|}{$\begin{array}{c}\text { Cuadro } \mathrm{N}^{\circ} 1 \\
\text { Comparación entre datos del mercado de trabajo y evolución } \\
\text { de la flexibilidad laboral (1989-1999) }\end{array}$} \\
\hline AÑo & MES & $\begin{array}{l}\text { Legislación } \\
\text { flexibilizadora }\end{array}$ & $\mid \begin{array}{ll}\text { Tasa de } \\
\text { Actividad }\end{array}$ & $\begin{array}{l}\text { Tasa de } \\
\text { Empleo }\end{array}$ & $\begin{array}{l}\text { Tasa de De- } \\
\text { socupación }\end{array}$ & $\begin{array}{l}\text { Tasa Subocu } \\
\text { pación (1) }\end{array}$ & $\begin{array}{l}\text { Porcentaje } \\
\text { de asalaria- } \\
\text { dos s/des- } \\
\text { cuento jubi- } \\
\text { latorio (2) }\end{array}$ \\
\hline \multirow[t]{2}{*}{1989} & Mayo & & 40,2 & 36,9 & 8,1 & 8,6 & $s / d$ \\
\hline & Octubre & & 39,3 & 36,5 & 7,1 & 8,6 & $s / d$ \\
\hline \multirow[t]{2}{*}{1990} & Mayo & & 39,1 & 35,7 & 8,6 & 9,3 & 27.0 \\
\hline & Octubre & 1 & 39,0 & 36,5 & 6,3 & 8,9 & 30.5 \\
\hline \multirow[t]{2}{*}{1991} & Junio & $\begin{array}{l}\text { Decreto sobre negocia- } \\
\text { ción productividad }\left(\mathrm{N}^{\circ}\right. \\
1334)\end{array}$ & 39,5 & 36,8 & 6,9 & 8,6 & 32.2 \\
\hline & Octubre & $\begin{array}{l}\text { Ley Nacional de Em- } \\
\text { pleo }\left(N^{\circ} 24.013\right)\end{array}$ & 39,5 & 37,1 & 6 & 7,9 & 34.3 \\
\hline \multirow[t]{2}{*}{1992} & Mayo & & 39,8 & 37,1 & 6,9 & 8,3 & 32.5 \\
\hline & Octubre & $\begin{array}{l}\text { Decreto sobre pasan- } \\
\text { tias }\left(N^{*} 340\right)\end{array}$ & 40,2 & 37,4 & 7 & 8,1 & 32.2 \\
\hline \multirow[t]{2}{*}{1993} & Mayo & $\begin{array}{l}\text { Decreto sobre negocia- } \\
\text { ción productividad }\left(\mathrm{N}^{\circ}\right. \\
470)\end{array}$ & 41,5 & 37,4 & 9,9 & 8,8 & 32.8 \\
\hline & Octubre & & 41,0 & 37,1 & 9,3 & 9,3 & 33.8 \\
\hline \multirow[t]{2}{*}{1994} & Mayo & & 41,1 & 36,7 & 10,7 & 10,2 & 31.7 \\
\hline & Octubre & & 40,8 & 35,8 & 12,2 & 10,4 & 33.5 \\
\hline \multirow[t]{2}{*}{1995} & Mayo & $\begin{array}{l}\text { Nuevo Régimen } \\
\text { Contratación ( } \mathrm{N}^{\circ} \\
44 . \\
465) \text { y Ley de PyMES } \\
\left(N^{\circ} 24.467\right)\end{array}$ & 42,6 & 34,8 & 18,4 & 11,3 & 34.1 \\
\hline & Octubre & & 41,4 & 34,5 & 16,6 & 12,6 & 35.5 \\
\hline \multirow[t]{2}{*}{1996} & Mayo & & 41,0 & 34,0 & 17,1 & 12,7 & 37.3 \\
\hline & Octubre & & 41,9 & 34,6 & 17,3 & 13,6 & 37,0 \\
\hline \multirow[t]{2}{*}{1997} & Mayo & & 42,1 & 35,3 & 16,1 & 13,2 & 37,1 \\
\hline & Octubre & & 42,3 & 36,5 & 13,7 & 13,1 & 37,8 \\
\hline \multirow[t]{2}{*}{1998} & Mayo & & 42,4 & 36,9 & 13,2 & 13,3 & 37,2 \\
\hline & Octubre & Ley de $\left(\mathrm{N}^{\circ} 25.013\right)$ & 42,1 & 36,9 & 12,4 & 13,6 & 37,9 \\
\hline 1999 & Mayo & & 42,8 & 36,6 & 14,5 & 13,7 & $\mathrm{~s} / \mathrm{d}$ \\
\hline
\end{tabular}

s/d: $\sin$ datos

(1) La tasa de subocupación es calculada como porcentaje entre la población subocupada y la población económicamente activa. Se consideran subocupados a todos los trabajadores que trabajan menos de 35 horas semanales por causas involuntarias y desean trabajar más horas. Para nues. tro trabajo hemos calculado el total de subocupados, sumando los que la EPH presenta como demandantes y no demandantes.

(2) El porcentaje de asalariados sin descuento jubilatorio se calcula a partir de los trabajadores que responden en forma negativa a la pregunta referida a si gozan de jubilación y representa en forma aproximada al conjunto de asalariados en situación de empleo no registrado.

Fuente: EPH-INDEC, Dirección Nacional de Relaciones de Trabajo-MTSS. 
produjo un leve descenso de la tasa de desocupación y un aumento en la tasa de actividad. Este leve impacto positivo en dichas variables no resulta significativamente importante si se toma en cuenta que dichas reglas fueron acompañadas por el shock de confianza que implicó la estabilización económica.

Respecto al porcentaje de asalariados sin descuento jubilatorio, alcanzaba al $34 \%$ en el momento de la puesta en marcha del sistema de regularización y la implementación de las modalidades promovidas de contratación de la Ley Nacional de Empleo. Un año después, quizás como producto de estas medidas se produce una disminución en dos puntos de dicho porcentaje.

En 1993 se produce un aumento importante de la tasa de desocupación, con una diferencia de tres puntos entre las ondas de mayo de este año y del año anterior. En esa misma medición se observa un incremento, aunque leve de la tasa de actividad, que se va a sostener por encima del $40 \%$ hasta la actualidad. En forma contraria a las explicaciones oficiales, este crecimiento no se debería a que el mercado de trabajo se presenta más atractivo, sino a la presencia de trabajadores sustitutos y complementarios (mujeres y jóvenes) del jefe de hogar, cuyos ingresos habían disminuido producto del desempleo o de la precarización. La tasa de empleo no muestra una pérdida de puestos de trabajo, ya que prácticamente se mantiene constante en estos dos años, pero no logra compensar el aumento de la actividad.

En los tres años que transcurren entre el ' 93 y el' 96 se produce un fenómeno sin precedentes en nuestro pais en re- lación a la problemática de la desocupación. En dicho periodo, este indicador sufre un aumento del $80 \%$, alcanzando el pico más alto en la medición de mayo 1995: la tasa asciende al $18,4 \%$, es decir que en solo dos años la desocupación se duplica (en términos absolutos equivale a aproximadamente 2.100 .000 personas en todo el país). Este hecho se ve acompañado y acrecentado por el descenso de la tasa de empleo en dos puntos. A su vez, los trabajadores en condiciones de precarización laboral, en el período tratado también aumentan su número.

Entre los años descriptos se sancionan una serie de leyes que apuntaban a facilitar la contratación de mano de obra y a disminuir el trabajo en negro (Nuevo Régimen de Contratación, Ley de PyMES, Decreto de Reducción de contribuciones patronales). Si consideramos que la reducción de los aportes patronales tendería a mejorar las condiciones de registrabilidad de los trabajadores ya que disminuyen los costos de seguridad social, esto debería haberse notado en un descenso del porcentaje de los trabajadores sin descuento jubilatorio, hecho que no se verifica entre los años 93-96. Asimismo, tomando en cuenta los datos vertidos más arriba en cuanto al aumento de las variables que caracterizan el mercado laboral, la legislación sancionada no contribuyó significativamente a revertir las tendencias observadas: por el contrario en ese mismo período tienden a aumentar.

En el mismo sentido podemos mencionar también, como aumento de la precarización laboral el crecimiento sostenido de la tasa de subocupación, que entre 1993 y 1996 subió aproximadamente cuatro puntos. 
Durante los dos años siguientes se experimentó una disminución en la tasa de desocupación, desde el 17\% de 1996 a aproximadamente el $13 \%$ en 1998 . La tasa de actividad, se mantuvo en valores cercanos al $42 \%$ en ese mismo periodo, lo que denota una presión muy grande sobre el mercado de trabajo. Al mismo tiempo, la tasa de subocupación trepaba a valores superiores al $13 \%$ y el porcentaje de asalariados sin descuento jubilatorio ascendia hasta el $37,9 \%$.

En octubre de 1998, el Poder Ejecutivo, después de enfrentamientos con el sector empresario y de la aceptación a regañadientes del FMI, logró la aprobación de la última ley flexibilizadora $\left(N^{\circ}\right.$ 25.013). Desde entonces hasta la medición de mayo de 1999, se produjo un aumento de la tasa de desocupación de dos puntos. Si bien este último valor puede ser atribuido a la última crisis brasileña, los instrumentos que el gobierno utiliza para lograr una contención del problema y asegurar un mejor funcionamiento del mercado de trabajo no parecen ser los más adecuados.

Las profundas transformaciones de la estructura productiva, los cambios en el paradigma de empresa y el aumento de sectores excluidos del mercado laboral obligaron al gobierno a la pronta implementación de pianes destinados a insertar a los trabajadores desocupados en el mercado de trabajo.

Así, desde el Ministerio del Trabajo y de la Seguridad Social se desarrollaron una multiplicidad de programas de empleo, que en su mayor parte constituyeron alternativas de muy baja calidad, absolutamente precarias, y con sueldos cercanos o por debajo del mínimo vital y móvil. Lo Vuolo (1998) caracteriza a estos programas por su multiplicidad, transitoriedad, baja afectación de recursos y limitada cobertura. Este comportamiento del Estado frente al crecimiento de la desocupación puede deberse a su relación contradictoria con la problemática. Una parte de sus políticas incentivan mecanismos expulsores o precarizadores de la mano de obra, mientras que otras deben destinarse al control de sus posibles consecuencias. En la mayor parte de los casos, se trata de programas que tratan de atender coyunturas especificas y se basan en un análisis, por lo menos superficial, de una realidad que aparece cada vez más complejo.

En el cuadro $\mathrm{N}^{\circ} 2$ podemos observar que el crecimiento en el número de beneficiarios en los programas de capacitación y empleo se produce en el momento en que el índice de desocupación toma valores más elevados (entre 1995 y 1997). De cualquier modo, en el año 1997, cuando dicho índice comienza a bajar, si se consideran en conjunto a los beneficiarios de empleo y capacitación sólo se llegaba a cubrir el $26 \%$ del total de desocupados ${ }^{11}$. Debemos destacar que el número de beneficiarios y la continuidad de los programas dependían, en gran

11 Es de destacar que un mismo individuo puede ser beneficiario del seguro de desempleo y estar inserto en un programa de capacitación o empleo. 


\section{Cuadro $\mathrm{N}^{\circ} 2$}

Trabajadores asistidos por programas del Ministerio de Trabajo y Seguridad Social (1992-1998)

\begin{tabular}{rrrc} 
& Programas de Capacitación & Programas de Empleo & Seguro de desempleo \\
\hline 1992 & & & 12.806 \\
1993 & 33.960 & 96.224 & 73.373 \\
1994 & 84.991 & 137.189 & 98.513 \\
1995 & 163.128 & 143.165 & 122.347 \\
1996 & 233.616 & 157.604 & 127.885 \\
1997 & 141.136 & 359.375 & 95.379 \\
1998 & 72.000 & 242.473 & 87.536 \\
Total & 728.831 & 1.136 .030 & 605.033 \\
\hline
\end{tabular}

Notas: No se incluyen los programas de empleo provinciales y de capacitación del Ministerio de Cultura Educación.

Fuente: Ministerio de Trabajo y Seguridad Social. Dirección Nacional de Políticas de Empleo y Capacitación Laboral.

parte, del financiamiento de organismos internacionales de crédito ${ }^{12}$.

Mientras tanto, el seguro de desempleo nunca llegó a cubrir un número considerable del total de trabajadores desocupados. Considerando que entre 1995 y 1996 se encontraban en esta situación más de 2 millones de personas, sólo aproximadamente el $6 \%$ de ellos contaban con este beneficio ${ }^{13}$.
Creemos importante destacar que la aplicación de políticas de empleo no parece haber tenido un efecto de atención y reducción de la problemática del desempleo y aún del empleo sin descuento jubilatorio. Al abarcar poca población del total de desocupados, estas politicas parecen tener una connotación propia del enfrentamiento de la coyuntura más que constituirse en una solución a la

12 El aumento de la tasa de desocupación puede tener relación directa con la emergencia de conflictos sociales en distintas zonas del país. El Estado, ante la necesidad de controlar la situación actúo mediante la inyección de políticas de asistencia destinadas a los sectores más desprotegidos. De cualquier modo, los recursos obtenidos redundaron en el aumento de la deuda asumida por el Estado con respecto a los Organismos Internacionales.

13 Debe aclararse que, según la legislación laboral argentina, existen algunas condicionamientos para el acceso al seguro de desempleo, ya que sólo está dirigido a los trabajadores bajo la LCT, es decir que no puede ser cobrado por aquellos que hayan desempeñado actividades en el sector agrario, servicio doméstico y la administración pública nacional, provincial o municipal. Además, entre otras restricciones, la Ley Nacional de Empleo indica que los trabajadores en las 
problemática. En este sentido, el bajo impacto de las políticas de empleo (en términos cuantitativos y cualitativos), puede deberse a políticas del estado sin proyección en el largo plazo con pretensiones de generar soluciones de fondo, y/o al exclusivo direccionamiento de muchas de ellas a la contención de focos de posibles estallidos sociales en los sectores más afectados por la desocupación y la pobreza.

\section{La flexibilidad en el servicio de telecomunicaciones y la industria automotriz}

\subsection{La negociación colectiva en el sector de telecomunicaciones}

En el sector de telecomunicaciones convergen una serie de fenómenos que por símismos hacen que se presente como un sector sumamente interesante para el estudio desde la perspectiva de las relaciones laborales. Por una parte, la empresa ENTel (Ente Nacional de Telecomunicaciones) fue la primera gran privatización realizada en Argenti$\mathrm{na}^{14}$. Proceso del que se extrajeron una serie de lineamientos que condicionaron las futuras privatizaciones ${ }^{15}$, y la forma cómo debían plantearse las relaciones laborales una vez que el Estado se desentendiera del gerenciamiento de las empresas de servicios públicos. Por otra parte, y desde la perspectiva productiva, las empresas de telecomunicaciones resultan ser relevantes ya que, en los últimos años, gozaron de una expansión sin precedentes debido a las innovaciones informáticas e informacionales.

condiciones anteriores deben haber efectuado cotizaciones al Fondo Nacional de Empleo durante un periodo mínimo de 12 meses en los tres años anteriores al cese del contrato. Esto último deja fuera del beneficio a los trabajadores con contratos temporarios de menor duración. Para los trabajadores contratados a través de empresas de servicios eventuales el período de cotización se reduce a 90 días durante los 12 meses anteriores al despido. Además, la disolución de la relación laboral debe haberse producido en los términos especificados por la LCT. Hay que tener en cuenta que cada vez más un gran número de trabajadores queda fuera de esta reglamentación por el crecimiento de relaciones laborales no registradas.

14 Si bien la privatización de Entel se llevó a cabo, no lue ciertamente sin una resistencia por parte del colectivo de trabajo que evaluó dicha situación como perjudicial a sus intereses desde una perspectiva coyuntural y de largo plazo.

15 La experiencia argentina en cuestión de privatizaciones de empresas públicas no tiene precedentes internacionales por su extensión y rapidez. Este agresivo programa tuvo como objetivos primordiales la reducción de las erogaciones del sector público y mejorar su flujo de caja; reducir el endeudamiento interno y externo a través del canje de titulos de la deuda por participaciones en la propiedad de las empresas privatizadas. 
La privatización de ENTel se llevó a cabo efectivamente en el mes de noviembre de $1990^{16}$ a través de una licitación internacional tras la cual la red nacional quedó dividida en dos zonas: norte y sur; proceso a través del cual Telecom Argentina y Telefónica de Argenti$\mathrm{na}^{17}$ accedieron a la concesión de dichos servicios.

En el período post-privatización, las negociaciones colectivas se dan en el contexto de una continua pérdida de puestos de trabajo (recordemos que entre el ' 90 y el '96 la caída redundó en 14.000 puestos) y una reducción consiguiente del número de posibles afiliados a las asociaciones sindicales. Esta situación contribuye a una reducción significativa del campo de acción de la organización obrera. Pero el poder de dicha organización se ve acotado por la presencia de sindicatos que disputan la representación de los trabajadores. Paralelamente a la Federación de Obreros y Empleados Telefónicos de la República Argentina (FOETRA $^{18}$, se presenta la Unión del Personal Jerárquico de Empresas de Te- lecomunicación (UPJET), la Federación de Organizaciones del Personal de Supervisión y Técnicos Telefónicos Argentinos (FOPSTTA), y el Centro de Profesionales Universitarios de las Empresas de Telecomunicaciones (CPTEL). Cada una de estas organizaciones firman Convenios Colectivos de Trabajo, atomizando de tal forma las estrategias sindicales en relación a la flexibilización laboral. Por otra parte, los sindicatos enrolados en la actividad de la construcción (UOCRA) y el Sindicato de Empleados de Comercio (SEC), también disputan el campo de acción pretendiendo para si la legitimidad en la representación sindical. Es llamativo, desde la perspectiva de la negociación que, mientras el actor sindical sufre una tendencia a la heterogeneización (en cuanto a la representación), el sector empresario en todos los casos firma Convenios Colectivos de Trabajo (CCT) por actividad, es decir las empresas "competidoras" asumen una estrategia unificada al momento de negociar.

En los CCT firmados a partir de 1991 se localizan diversos aspectos flexi-

16 Un primer intento de privatizar el área de telecomunicaciones se llevó a cabo bajo el gobierno de R. Alfonsín, de la mano del entonces Ministro de Obras y Senvicios Públicos R. Terragno, por el cual se proponía la incorporación del $40 \%$ de capital privado a ENTel.

17 Telecóm Argentina está conformada en forma mayoritaria por capitales franceses e italianos y Telefónica de Argentina mayormente por capitales españoles. Los servicios de larga distancia internacional quedó a cargo de la empresa Telintar; los servicios de telex, transmisión de datos, telefonía móvil y radio móvil marítimo, bajo la órbita de la empresa Startel. En ambos emprendimientos los dos consorcios comparten el capital accionario.

18 El sindicato modificó su denominación a fines de 1996 pasando a llamarse FOEESSITRA (Federación de Obreros, Especialistas y Empleados de los Servicios e Industria de las Telecomunicaciones de la República Argentina. La nueva denominación del sindicato responde a la necesidad de acoger a otros trabajadores que antes no estaban contemplados. 
bilizadores, entre los cuales podemos mencionar la implementación de la movilidad o polivalencia funcional en las tareas cotidianas de los trabajadores (CCT $N^{\circ} 201 / 92$ ).

Asimismo, en el CCT N ${ }^{\circ}$ 257/97E se plantea que los trabajadores deberán aportar en forma concurrente y simultánea o alterna todos los conocimientos y habilidades correspondientes a sus capacidades. La apropiación de ese saber hacer pretende sistematizar los saberes adquiridos en la práctica; $y$ en última, instancia favorece la productividad aportada por el conocimiento de las problemáticas surgidas en el proceso productivo cotidiano y en el modo que estos son resueltos.

Una idea complementaria a la polivalencia es la referencia que se realiza en algunas convenciones colectivas a los denominados grupos de trabajo o grupos laborales con movilidad funcional (CCT N² 257/97). En términos generales estas cláusulas se vinculan con la transmisión de conocimientos que, a través de los cursos de capacitación se realizan en la empresa. "La oferta de cursos es flexible en ambas licenciatarias pues evoluciona en función de la readecuación de los programas en relación a la introducción de nueva tecnología y a la intención de ambas empresas de inducir al personal a adoptar nuevos valores (en particular los relacionados con la productividad y la calidad del servicio), en un contexto signado, como consecuencia de la tradicional desvinculación entre el sistema educativo argentino y el sector productivo en el área de telecomunicaciones, por importantes carencias en cuanto a la oferta educativa" (Walter y Senen González, 1998).
Las referencias expuestas se encuentran vinculadas con la reorganización del trabajo al interior de la empresa, en este sentido el CCT N ${ }^{\circ}$ 257/97E deja abierta la posibilidad a introducir todos aquellos cambios relativos a la forma y modalidades de prestación de servicio.

En algunos convenios del sector se hace explicita referencia a la necesidad de encontrar mecanismos que disuelvan el conflicto en la empresa. En otros se utiliza la fórmula de la paz social para diferir la expresión de la conflictividad, CCT ${ }^{\circ}$ 201/92.

En todos los casos se hace referencia para la determinación de posibles actitudes conflictivas, a la necesidad de la prestación de un servicio y la adecuada atención al cliente. Así, la relación entre capital y trabajo aparece mediatizada por el cliente, que actúa como cuña entre ambos actores.

Por otra parte, las convenciones colectivas suscritas modificaron ciertos subsidios o beneficios convencionales para la actividad, produciéndose una pérdida de los beneficios salariales indirectos. De este modo, "las reducciones se inician con el Decreto $1757 / 89$, que establece la eliminación de beneficios que inciden sobre la productividad y eficiencia de la empresas públicas. La pérdida de la garantia del empleo propia del estatuto de la función pública y la abolición de beneficios específicos del sector tales como el descuento del $75 \%$ de la tarifa telefónica mensual y la instalación y el cambio de titular sin cargo, que desaparecieron de los convenios firmados en 1991 y 1992" (Walter y Senen González, 1998).

En el Art. 70 del CCT N² 257/97 se establece el cómputo correspondiente a la 
licencia por vacaciones sobre la base de días corridos, cuando en los convenios firmados en el periodo pre-privatización se refería a días hábiles. Vinculado a este aspecto, en el mismo convenio se hace referencia a la posibilidad del otorgamiento de las vacaciones en cualquier momento del año e incluso su fraccionamiento.

A su vez, en el mismo Artículo 70 se habilita la posibilidad de fraccionar las vacaciones:

"Cuando el periodo vacacional adquirido supere los catorce (14) días corridos y conforme las necesidades del servicio, La Empresa podrá programar y otorgar las vacaciones en forma fraccionada colectiva y/o individual en dos épocas del año"

De tal forma, se busca reducir los costos y mejorar el uso de la capacidad instalada adaptando la mano de obra a la necesidad operativa de la empresa.

En las relaciones de hecho, establecidas en las empresas telefónicas, se planteaba una resistencia de los trabajadores a acceder a sus vacaciones, por temor a perder el puesto o que su tarea sea desempeñada por otros trabajadores. Indudablemente, en este caso el alto índice de desempleo actúa disciplinando la mano de obra, vehiculizando e incentivando la implementación de factores flexibilizadores más allá de lo institucionalizado a través de las convenciones colectivas.

Es necesario resaltar, además, que se introducen cláusulas que favorecen la predisposición de los trabajadores a intensificar el trabajo por medio de premios diferenciales:
"Premio por productividad: Cada empleado Jerárquico percibirá semestralmente, su valor equivale al $50 \%$ del sueldo del mes y del valor antigüedad en que corresponda abonarlo".

El aumento de la productividad es incentivado en la misma empresa mediante el reconocimiento simbólico de la "mayor predisposición al trabajo".

Por último, los convenios firmados facilitan la entrada y salida de trabajado. res en tanto habilitan las formas contractuales promovidas $\mathrm{y}$ otras modalidades que apuntan a aumentar el plantel bajo contratos por tiempo determinado, contrabalanceando aquellos puestos de trabajo bajo la histórica relación indeterminada en el contrato de trabajo.

Un síntoma muy importante de la flexibilización laboral en la empresa es la utilización de contratos de pasantía en puestos de atención al cliente. Se pone de manifiesto así una contradicción entre la manifestación empresaria de garantizar una adecuada atención y mejorar las relaciones con el cliente y la utilización de mano de obra precaria, con bajas remuneraciones y débil implicación en el pues. to de trabajo. Además, la inadecuación entre la formación previa de los pasantes y la tarea requerida hace que queden inhibidas las intenciones originales orientadas y basadas en la idea que formas de este tipo podrían contribuir a la capacitación de los alumnos próximos a insertarse a nivel profesional.

\subsection{La negociación colectiva en el sector automotriz}

En el sector automotriz la negociación colectiva formalizada y homologada 
ante las autoridades administrativas presenta características especiales. Por un lado aparece como paradigmática de la flexibilización laboral y por el otro presenta rigideces típicas del modelo productivo anterior.

Algunos convenios colectivos firmados por el Sindicato Metalmecánico Argentino del Transporte Automotor (SMATA) con distintas empresas fueron tomados como ejemplo por sectores empresarios e incluso por el mismo Estado acerca de cuales deben ser las cláusulas que deben ser introducidas para lograr una modernización de las relaciones laborales en las empresas ${ }^{19}$.

A través de estas convenciones se disponen mecanismos que refuerzan la disciplina en la empresa y aseguran una correlación muy fuerte entre los intereses y la implicación de los trabajadores en la producción. Así, se distinguen cláusulas relativas a: la concreción de las licencias convencionales debe ser acordada al interior de cada célula para no afectar los objetivos del grupo; y la obligación para los delegados de coordinar el abandono de sus tareas con sus colegas de célula y obtener la aprobación del responsable de la manufactura o de célula. Se indican, además, mecanismos para evitar los conflictos en la empresa. (CCT N ${ }^{\circ}$ 98/94 de General Motors).

Con el mismo objetivo anterior y aumentando las presiones sobre los trabajadores se disponen incentivos monetarios basados en la productividad, la atención al cliente, la venta de unidades y la calidad del producto. Este tipo de dispositivos tiende a incrementar la competencia entre los trabajadores al interior del grupo o entre distintos grupos, con el objetivo de obtener dichos incentivos. Además, se tiende a un autodisciplinamiento de los trabajadores que pueden dejar de lado solidaridades de grupo. Pueden generarse situaciones en que los trabajadores estén dispuestos a sacrificar sus descansos o ser incentivados a permanecer en la empresa aun aquejados por alguna enfermedad, con el consiguiente perjuicio para su salud. Esto puede agravarse con la intensificación del trabajo que se produce al realizar tareas bajo la presión

"La negociación por empresa en el sector se construye a partir de la propuesta de las terminales de impulsar sindicatos 'de' empresa. La acción sindical transforma esta propuesta en una negociación 'por' empresa. Esta es una diferencia de trascendental importancia a la hora de entender las estrategias del sindicato automotriz. El modelo de 'Sindicato de empresa' a la manera japonesa remite a un sistema de relaciones laborales que se maneja con exclusividad en el interior de la planta u organización productiva. El sindicalismo 'por empresa' remite a un sistema de relaciones laborales que, aunque con capacidad de negociar acuerdos en el interior de la empresa, responden a la figura del sindicato de rama o sector del cual dependen" (Novick y Catalano, 1996). Los convenios firmados por el SMATA fueron acordados por la comisión interna de la empresa y el sindicato nacional y/o regional con las empresas, en cambio la UOM aún no ha modificado su convenio por actividad $\left(N^{\circ} 260 / 75\right)$ y sus dirigentes sostienen su negativa a negociar a nivel de la planta un nuevo convenio. 
de la obtención de un objetivo y a través de él un salario más elevado.

Por otra parte, la incorporación de múltiples componentes variables en el salario hace que el trabajador pierda la dimensión del dinero que dispone al finalizar cada uno de los meses en que transcurre la relación salarial. La inseguridad actúa como incentivo a la búsqueda del compromiso con la producción y puede potenciar el abandono de los tiempos propios del disfrute personal.

Los convenios más modernos firmados por el Sindicato Metalmecánico Argentino del Transporte Automotor. (SMATA) establecen a la polivalencia y la polifuncionalidad como formas de trabajo funcional a las nuevas caracteristicas de la organización del trabajo. Trabajadores más implicados y con disponibilidad para realizar cualquiera de las tareas requeridas en el interior del grupo 0 aún de distintos grupos responden de forma más eficiente a las necesidades de la producción. Estas metodologías permiten a la empresa una reducción importante en la demanda de personal, ya que se cuenta con la mano de obra necesaria para responder a fluctuaciones de la producción y a los distintos momentos de la organización.

Los trabajadores o sus células pasan a ser considerados como clientes internos de otros trabajadores o células. La denominación cambia el sentido de pertenencia del trabajador y sus relaciones con el resto del colectivo obrero. Ser cliente significa una referencia externa y diferenciada respecto al otro, el cliente no es un "igual" sino un "demandante" al que hay que responder y no con el que hay que compartir problemas de la produc- ción. El cliente requiere soluciones y respuestas a sus demandas, que suelen ser urgentes y de mucha exigencia. Los trabajadores se ven así presionados por la urgencia de la producción y por sus propios compañeros.

Esta relación deviene impropia, el trabajador es obligado en muchos casos a relacionarse con sus propios compañeros sobre la base de la prestación de un servicio al otro. Las necesidades de mantener una buena atención al cliente se transmiten al conjunto de los trabajadores, desviando así de su atención las problemáticas propias de la relación laboral.

Las capacidades para relacionarse de esta manera pueden ser transmitidas a los trabajadores por la misma empresa. En muchos casos, los círculos de calidad, los cursos de capacitación y las consignas difundidas por los departamentos de recursos humanos suelen ir en ese sentido. Tal como lo indica Gorz (1997): "La profesionalización de las "competencias relacionales", como medio de desarrollar el empleo, envenena y atrofia la cultura del cotidiano y el arte de vivir".

En varios de los convenios firmados por SMATA a partir de 1994 (General Motors; lochpe-Maxion; Toyota; Yasaki; Chrysler S.A.; Johnson Controls) se opta por la modularización de la jornada en forma anual. Se instala así un mecanismo para disponer de mejor manera la utilización de la mano de obra, en función de las necesidades de la producción. Este dispositivo puede resultar en formas de utilización del tiempo en que las urgencias de la empresa disuelvan el tiempo propio de los trabajadores. Según la misma gerencia de Recursos Humanos de una de estas empresas, jamas se llegó a utilizar el total de 
horas pactadas en el convenio durante el año. Entonces, la pregunta puede surgir en forma inmediata: ¿para qué lo negociaron? ¿no será que de esta forma se aseguran la disponibilidad de sus trabajadores en cualquier momento del año, el tiempo que la producción lo requiera?

Se introdujeron además las modalidades de contratación promovidas, que permitían la utilización de mano de obra en forma temporaria y para requerimientos específicos. En los convenios posteriores a 1995 se aumenta a 6 meses el período de prueba (CCT N ${ }^{\circ}$ 185/95: SMATA C/FIAT AUTO; CCT N ${ }^{\circ}$ 188/96: SMATA CMAGNETTI MARELLI DENSO; CCT N ${ }^{\circ}$ 189/96: SMATA $C / C H R Y S L E R ;$ CCT N ${ }^{\circ}$ 190/96: SMATA $C$ /TOYOTA), tal como lo preveía la Ley $N^{\circ} 24.465$. Debe destacarse que dicho período puede ser utilizado como una nueva forma de contratación temporaria, dada la posibilidad de contar con un trabajador por un período corto y sin tener obligaciones de realizar las contribuciones a la seguridad so$\mathrm{cial}^{20}$ y pagar la indemnización correspondiente al finalizar el mismo.

En algunos convenios se fraccionan las vacaciones en dos periodos, para aquellos casos que superen los 14 dias, y se las otorga entre los meses de diciembre y febrero y entre julio y agosto (CCT No 185/96: SMATA C/IOCHPEMAXION Argentina S.A.; CCT $\mathrm{N}^{\circ}$ 188/96: SMATA C/MAGNETTI MARELLI DENSO). Debe recordarse que la licencia anual es un derecho de los trabajadores, por el cual logran un período de tiempo en el cual se interrumpe su relación laboral y su dependencia respecto del empleador. Se trata de un tiempo propio, en el que no se trabaja y el trabajador adquiere un estatus similar al del empleador (CASTEL, 1995). Si se considera que este tiempo es propiedad individual del trabajador, debe estar a su disposición y no debe ser otro el que determine cuando y como se utiliza.

Cuando se describen los perfiles de los trabajadores requeridos se hace referencia a la necesidad de que estos realicen tareas de calidad como complementarias a la actividad correspondiente a sus calificaciones y su puesto de trabajo. Esto contribuye a la reducción o eliminación directa del área específica de la empresa, pero por otra parte se trasladan responsabilidades a los trabajadores que deben ahora estar preparados y atentos ante cualquier defecto o problema de la producción.

En el convenio de FIAT AUTO (CCT N $\left.{ }^{\circ} 185 / 96\right)$ se prevé una disminución del $25 \%$ en los salarios de los trabajadores en el caso de rotura, limpieza de máquinas, falta de materias primas u otro motivo no imputable al trabajador. Los riesgos de la producción dejan de ser asumidos en forma plena por el dueño del capital. Las contingencias especiales, producto incluso del mismo desgaste de las máquinas, son atendidas con una porción del salario sin afectar así la tasa de ganancia.

Las tareas de liderazgo se derivan en algunos componentes de la célula, ca- 
paces de tomar decisiones y de dirigir el desarrollo de las actividades internas. De este modo, se achata la pirámide de control en la empresa, ya que no se requiere de puestos de supervisores en cada uno de los sectores de la misma. Pero, además se consigue disponer un control mucho más cercano a los trabajadores cuyas tareas son seguidas por un "igual" en forma continuada. Se establece además una competencia por la representación de los trabajadores entre el sindicato y estos nuevos trabajadores. "El reclutamiento de líderes por parte de la empresa, entre los trabajadores con más capacidad, iniciativa, ambiciones, arraigo y carisma, produce -o puede producir-, por otra parte, un vaciamiento de potenciales figuras sindicales. A la larga, la organización sindical, si perdura, se quedaria sin relevos con capacidad significativa" (Stolovich y Lezcano, 1996).

Es importante destacar que este tipo de negociaciones fueron llevadas a cabo por el Sindicato de Transporte Automotor (SMATA) en empresas de reciente instalación en el país, y en algunos casos antes que se hayan efectivizado los contratos con los trabajadores. En los casos de algunas empresas ya instaladas en el país, el gremio negoció sus convenios en la ronda iniciada en 1988, sin introducir mayores modificaciones flexibilizadoras y siguiendo las características de las negociaciones de los años '70. Entre 1988 y 1989, el SMATA discutió con los empre. sarios un total de 33 convenios, en los cuales "las nuevas pautas incorporadas no revelan cambios profundos del sistema de organización del trabajo. Las demandas parecen basarse en la actualización de un sistema tradicional que tenia las negociaciones congeladas desde hace trece años. Sin embargo, se produjeron cambios en las industrias terminales que los convenios no reflejan" (Novick, Catalano, 1996) ${ }^{21}$.

En el caso particular de un contrato colectivo firmado con la empresa DEUTZ, en el año 1989, el gremio se niega a la renovación del mismo, aún después de que la empresa haya sido transferida a nuevos dueños (AGCO de capitales estadounidenses y posteriormente CARRARO de capitales italianos). La comisión interna de la empresa asegura que el convenio vigente les permite impedir la introducción de flexibilidades y la eliminación de los derechos alcanzados en el momento de su negociación.

De cualquier modo, "a pesar de no figurar en los convenios, las empresas líderes del sector automotriz presentan actualmente una multiplicidad de innovaciones organizacionales", entre las que se pueden destacar: gestión de la producción bajo lógicas de producción ligera (Lean Production), subcontratación y externalización del proceso productivo, reducción de stocks, implementación de programas de Mejora Continua, cambios en el área de mantenimiento y reorgani-

21 Algunos ejemplos de esta situación se presentan en los casos de los convenio firmado por SMATA con las firmas DEUTZ ( $N^{\circ} 3 / 89$ ), AUTOLATINA ( $N^{\circ} 8 / 89$ ), MERCEDES BENZ ( $N^{\circ} 14 / 89$ ), RENAULT ( $N^{\circ} 15 / 89$ ); SAAB ESCANIA ( $\left.N^{\circ} 26 / 90\right)$. 
zación de los puestos de trabajo tendiente a constituir módulos o células de trabajo. (Bisio y Freytes Rey, 1998).

De esta manera, el comportamiento sindical estaría asemejándose al de la Unión Obrera Metalúrgica (UOM), que mantiene sin modificaciones el convenio firmado en 1975 ( $\left.N^{\circ} 260 / 75\right)$, tratando de evitar de este modo modificaciones que vayan en contra de las protecciones alcanzadas.

En este último caso, la UOM, al contrario de la práctica del Sindicato de Transporte Automotor (SMATA), permanece en su postura contraria a la negociación por empresa. A pesar de esto, y quizás como consecuencia de la necesidad que tuvieron las comisiones internas y las seccionales de responder a las presiones impuestas por las transformaciones productivas, se negociaron a nivel de empresa bajo la forma de "actas acuerdo", nuevas formas de la organización del trabajo a cambio de aumento en las remuneraciones. Se produce asi una descentralización de hecho en la negociación y una falta de articulación entre las distintas seccionales y la conducción central, producto de la negativa a negociar por parte de esta última (Bisio y Freytes Rey, 1998).

El éxito de la conducción central de UOM en el mantenimiento de condiciones impuestas por el convenio de 1975 parece ser relativo, ya que sólo se logró a nivel de lo formal. Sin embargo, si se considera que los derechos se concretan en la norma, su disolución definitiva terminaría de concretarse en el momento en que el convenio sea dejado de lado.

\section{Conclusión}

Tal como pudimos observar, en nuestro país la decisión de flexibilizar las relaciones de trabajo implicó un retroceso muy importante en las protecciones a los trabajadores, sin que como contrapartida se haya producido un mejoramiento substancial en los indicadores del mercado de trabajo. El aumento en la tasa de desempleo $y$ en la precarización laboral fue el signo de toda la época.

En la legislación, la opción primera estaba dirigida a la implementación de mecanismos relacionados con to que se denomina flexibilidad externa. Sin embargo, dadas las ambivalencias y contradicciones mostradas por el gobierno, no siempre las reformas fueron dirigidas en el mismo sentido. En cada uno de los casos pareció responderse a presiones de los sectores empresariales y/o sindicales, dependiendo de la coyuntura económica y política vigente.

La adecuación de las relaciones laborales a la realidad productiva podia obtenerse entonces mediante la vía negocial. La debilidad de los sindicatos $y$, en muchos casos, la necesidad de responder a las presiones empresariales derivó en un retroceso muy importante de las conquistas obreras. Algunos de los convenios firmados, desde mediados de los años 90 , reflejan la imposición de un marco cultural ajeno a los trabajadores, más relacionado con las necesidades de las nuevas formas de organización del trabajo y gestión de la mano de obra. En otros casos, las organizaciones sindicales lograron mantener en vigencia los convenios firmados bajo el marco del régimen 
de acumulación basado en el modelo de industrialización por substitución de importaciones, cuando su posición en la relación de fuerzas les había permitido obtener conquistas importantes para los trabajadores. De cualquier manera, la realidad en las empresas parece contrastar con el marco que presentan estos viejos convenios y la introducción de la flexibilidad no parece encontrar demasiados obstáculos.

Para tratar de responder al aumento de la tasa de desempleo y controlar la precarización creciente que se instalaba en el mercado de trabajo se ensayaron políticas que actuaron sólo como paliativo para los sectores más desprotegidos, sin constituirse en verdaderos instrumentos destinados a la reconversión y reinserción de los trabajadores.

El endeudamiento estatal que se producía entre los años 40 y 70 , tenía entre sus objetivos, el incremento de la protección del trabajo, que redundaría en incrementos de la demanda de productos y senvicios. Durante el gobierno de Menem, el Estado se endeudó para cubrir sólo a una mínima porción de los excluidos, después de haber pasado por un proceso de achicamiento y privatización desarrollado con el objetivo de disminuir la deuda antes contraída. Ante el aumento de la violencia cotidiana, las políticas de empleo, tanto activas y pasivas, así como el desarrollo de instrumentos de política social complementarios, pareció la única opción, pero genera un crecimiento sostenido del endeudamiento externo. Sin embargo, si estas políticas se hubieran articulado en forma coherente con las que se dirigía a la concreción de un mo- delo de industrialización de tipo mano de obra intensivo y con políticas educativas que tendieran a potenciar las capacidades técnicas y científicas del sistema productivo en su conjunto, podrian haberse esperado mejores resultados en el mediano o largo plazo.

\section{Bibliografía citada}

Battistini, O. y Montes Cató, J. (1998). "¿Flexibilidad negociada? El dilema de la elección del nivel", ponencia presentada en el IV Congreso Nacional de Especialistas en Estudios del Trabajo. noviembre.

Battistini, Osvaldo R. (1998). "Un entoque alternativo para el análisis del sistema de relaciones laborales", ponencia presentada en el IV Congreso Nacional de Especialistas en Estudios del trabajo, noviembre, Buenos Aires.

Battistini, O; Jabbaz et. al (1996). "La negociación colectiva: un vehiculo para la flexibilidad labora" ", trabajo presentado en el ll Congreso Latinoamericano de Sociologia del Trabajo, Rlo de Janeiro, diciembre.

Bisio, R. y Freites Rey, A. (1998). "Nuevos escenarios de negociación laboral. UOM vS. SMATA: ¿divergentes planteos estratégicos de acción sindical?", mimeo.

Boyer, A. (1987), "La flexibilidad laboral en Europa", Ministerio de Trabajo y Seguridad Social, Madrid.

Boyer (1989). La teoria de la regulación. Un análisis critico, Ed. HVMANITAS, Buenos Aires.

Brinkman, M. E. (1999). "Las relaciones laborales en el sector telefónico", en Fernández, A. A. y Bisio, R.A H. (comps.) Política y Relaciones Laborales en la transición democrática Argentina, Ed. Lumen Humánitas, Buenos Aires. 
Castel, Robert (1995). Las metamortósis de la cuestión social, Paidos, Buenos Aires

Catalano, A.; Hernández, D. y Rojas, E. (1992). "Crisis, Flexibilidad y Acción Colectiva", En Revista Justicia Social, Año 7, № 11/12, Buenos Aires.

Deibe, E., Matheu, P. y Estévez, A. (1994). "Productividad y negociación laboral en Argentina 1991/1994", ponencia presentada al $2^{\circ}$ Congreso Nacional de Estudios del Trabajo, ASET, Buenos Aires.

Gorz, André (1997). Misères du present. Ai* chesse du possible, Ed. Galilée, Paris.

Lo Vuolo, A. (1998). "Crisis de integración social y retracción del Estado de Bienestar en Argentinan ${ }^{n}$, en Lo Vuolo y Barbeito (1998). La nueva oscuridad de la politica social. Del Estado populista al neoconservador, Niño y Dávila Edito. res, Buenos Aires.

Novick, M. y Catalano, A. (1996). "Industria Automotriz Argentina: Redefiniendo Estrategias Productivas, Mercados y Relaciones Laborales", en Los Efectos Laborales de la Reestructuración Productiva, Universidad de $\mathrm{Ca}$ - rabobo, Héctor Lucena Ed., Valencia, Venezuela.

Santantonio, S. y Tavilla, P. (1995). "Flexibilización laboral, empleo y productividad: aportes para debatir el caso argentino", Documento de Trabajo №1, PIETTE-CONICET, Buenos Aires.

Spyropoulos, G. (1991). Sindicalismo y Sociedad, Ed. Humánitas, Buenos Aires.

Stolovich, L. y Lescano, G. (1996). "El desafio de la calidad total o cuando los sindicatos se sienten atacados", en Revista Latinoamericana de Estudios del Trabajo, Año $2 N^{\circ} 4$, ALSTRA, México.

Walter, J y Senen González (1996). "Modos de modernización tecnológica y movilización de los recursos humanos en las empresas argentinas privatizadas", en Revista Dialógica, Nủmero Especial, Vol 1, No1, CEIL-CONICET, Buenos Aires.

Walter, J y Senen González comps. (1998). Las privatización de las telecomunicaciones en América Latina. Empresas y sindicatos ante los desafios de la reestructuración, Ed. Eudeba, Buenos Aires. 